\title{
CORRECTION
}

\section{Correction to: Renal histology across the stages of chronic kidney disease}

\author{
Francesco Trevisani ${ }^{1,2}$. Federico Di Marco ${ }^{2}$ Umberto Capitanio ${ }^{1,2}$. Giacomo Dell'Antonio ${ }^{3}$. Alessandra Cinque ${ }^{2}$. \\ Alessandro Larcher ${ }^{1,2} \cdot$ Roberta Lucianò $^{3} \cdot$ Arianna Bettiga $^{2} \cdot$ Riccardo Vago $^{2}$ - Alberto Briganti ${ }^{1,2} \cdot$ Andrea Salonia $^{1,2}$. \\ Francesco Montorsi ${ }^{1,2} \cdot$ Esteban Porrini ${ }^{4}(0)$
}

Published online: 13 May 2021

(c) Italian Society of Nephrology 2021

\section{Correction to: Journal of Nephrology} https://doi.org/10.1007/s40620-020-00905-y

While typesetting the article, the full author names are not Publisher's Note Springer Nature remains neutral with regard to included. The correct author names are provided below.

Francesco Trevisani

Federico Di Marco

Umberto Capitanio

Giacomo Dell'Antonio

Alessandra Cinque

Alessandro Larcher

Roberta Lucianò

Arianna Bettiga

Riccardo Vago

Alberto Briganti

Andrea Salonia

Francesco Montorsi

Esteban Porrini

The original article has been corrected.

The original article can be found online at https://doi.org/10.1007/ s40620-020-00905-y.

Esteban Porrini

esteban.1.porrini@gmail.com

1 Unit of Urology, IRCCS San Raffaele Scientific Institute, Milan, Italy

2 Urological Research Institute (URI), Division of Experimental Oncology, IRCCS San Raffaele Scientific Institute, Milan, Italy

3 Unit of Pathology, IRCCS San Raffaele Scientific Institute, Milan, Italy

4 Facultad de Medicina, ITB: Instituto de Tecnologías Biomédicas, Universidad de La Laguna, 38320 La Laguna, Spain 